\title{
Erratum to: Development of SSR markers and assessment of genetic diversity of adzuki bean in the Chinese germplasm collection
}

\author{
Honglin Chen • Liping Liu • Lixia Wang • \\ Suhua Wang • Ming Li Wang • Xuzhen Cheng
}

Published online: 2 May 2017

C) Springer Science+Business Media Dordrecht 2017

\section{Erratum to: Molecular Breeding \\ DOI $10.1007 / \mathrm{s} 11032-015-0383-5$}

The original version of this article contained text from previously published papers without giving the proper references. Below are the affected texts with correct acknowledgment of references.

Introduction

However, the number of SSR markers reported (Chen et al. 2015a) for adzuki bean is still far fewer than the one from other legumes such as

The online version of the original article can be found at http://dx. doi.org/10.1007/s11032-015-0383-5

H. Chen $\cdot$ L. Wang $\cdot$ S. Wang $\cdot$ X. Cheng $(\bowtie)$

National Key Facility for Crop Gene Resources and Genetic Improvement, Institute of Crop Science, Chinese Academy of Agricultural Sciences, Beijing 100081, China

e-mail: chengxuzhen@caas.cn

L. Liu

State Key Laboratory of Agrobiotechnology, College of

Biological Sciences, China Agricultural University,

Beijing 100193, China

M. L. Wang

United States Department of Agriculture, Agricultural Research Service, Plant Genetic Resources Conservation Unit, Griffin, GA 30223, USA mung bean (Chen et al. 2015b), common bean (Blair et al. 2011), chickpea (Choudhary et al. 2009), pigeon pea (Dutta et al. 2011) and soybean (Xin et al. 2012; Zhang et al. 2013).

Materials and methods

400 eSSR targeting primer pairs (Supplement Table 2) were randomly selected and designed using Primer 3.0 based on the following criteria: primer length between 18 and $22 \mathrm{bp}$, product sizes between 100 and $300 \mathrm{bp}$ and with a GC content of 40$60 \%$ (Chen et al. 2015a). A total of 100 gSSRs were designed with the same criteria as described above based on the genomic sequence of mung bean available under GenBank/EMBL/DDBJ accession code JJMO00000000 (Kang et al. 2014).

Population structure was assessed using STRUCTURE 2.34 (Pritchard et al. 2000). The range possible of K-values was from 2 to 15 , with ten independent runs each and 100,000 Markov Chain Monte Carlo (MCMC) iterations. We chose the most likely number of clusters by plotting the $\mathrm{LnP}(\mathrm{D})$ values against $\Delta \mathrm{k}$ values and the best $\mathrm{K}$ value based on the rate of change of the logarithm of probability (Evanno et al. 2005). The genetic structure of adzuki bean accessions was further determined by analysis of molecular variance (AMOVA) using GenAlEx 6.5 (Peakall and Smouse 2012; Chen et al. 2015b). 
Result

Under category of the biological process cellular process $(16.1 \%)$ and metabolic process $(15.9 \%)$ were the most abundant subcategories, followed by metabolic process (15.9\%), and single-organism process $(10.8 \%)$. In the category of cellular component, cell $(25.2 \%)$ and organelle $(19.9 \%)$ represented the most abundant subcategories. However, only a few unigenes were assigned to extracellular matrix $(<0.1 \%)$ and virion $(<0.1 \%)$. The molecular function category was mostly dominated by catalytic activity (42.1\%) and binding activity (42.0\%) (Chen et al. 2015a).

\section{References}

1. Chen H, Wang L, Wang S, Liu C, Blair M, Cheng X (2015a) Transcriptome sequencing of mung bean (Vigna radiate L.) genes and the identification of EST-SSR markers. PLoS One 10(4):e0120273

2. Chen H, Liu L, Wang L, Wang S, Somta P, Cheng X (2015b) Development and validation of ESTSSR markers from the transcriptome of adzuki bean (Vigna angularis) PLoS One 10(7):e0131939

3. Pritchard JK, Stephens M, Donnelly P (2000) Inference of population Structure using multilocus genotype data. Genetics 155:945-959 